\title{
A clinical study on the use of Huaier granules in post-surgical treatment of triple-negative breast cancer
}

\author{
Minghao Wang ${ }^{1 \#}$, Ying Hu ${ }^{1 \#}$, Lingmi Hou ${ }^{2 \#}$, Qinwen Pan $^{1}$, Peng Tang ${ }^{1}$, Jun Jiang ${ }^{1}$ \\ ${ }^{1}$ Breast Disease Center, Southwest Hospital, Army Medical University, Chongqing 400038, China; ${ }^{2}$ Department of Thyroid and Breast Surgery, \\ Affiliated Hospital of North Sichuan Medical College, Nanchong 637000, China \\ Contributions: (I) Conception and design: J Jiang; (II) Administrative support: P Tang; (III) Provision of study materials or patients: M Wang; (IV) \\ Collection and assembly of data: L Hou; (V) Data analysis and interpretation: Y Hu, Q Pan; (VI) Manuscript writing: All authors; (VII) Final \\ approval of manuscript: All authors. \\ \#These authors contributed equally to this work. \\ Correspondence to: Prof. Peng Tang; Prof. Jun Jiang. 30\# Gaotanyan street Chongqing 400038, China. \\ Email: southwestbreast5@163.com; southwestbreast@163.com.
}

Background: In recent years, traditional Chinese medicine (TCM) has been developing rapidly in cancer treatment. Huaier is a widely used fungus by TCM to treat different kinds of cancers. Its good efficacy in prevention of tumor recurrence and metastasis has been proven by a large number of clinical studies. In order to further investigate the efficacy and safety of Huaier granules in post-surgical therapy for stage IIII triple-negative breast cancer (TNBC) patients, we performed a case-control clinical study to observe its effects on the post-surgical safety and survival rates of these patients.

Methods: Two hundred and one TNBC patients underwent modified radical mastectomy were selected, they were admitted to our hospital between October 2010 to September 2014. The patients were randomly allocated to the experimental group (101 cases) or the control group (100 cases). Patients in the experimental group were treated with Huaier granules, by orally taking $20 \mathrm{~g}$ each time with 3 times a day. Medication was started during chemotherapy or at the time in 6 or 18 months after it. The control group did not receive any TCM preparations during this process. The disease-free survival (DFS) and overall survival (OS) were measured as the main outcome.

Results: The median follow-up time was 46 months. For the 100 patients in control group, 5-year DFS and OS was $82 \%$ and $86 \%$ respectively, while $87.1 \%$ and $90.1 \%$ for the 101 patients in the experimental group. The difference was not statistically significant. However, stage III patients in the control group showed a 5 -year DFS of $53.8 \%$ and OS of $65.4 \%$, which were significantly lower than that of stage III patients in the experimental group as $81.3 \%$ and $87.5 \%$. In the experimental group, 10 patients with 6 -month medication showed disease progression, whereas only 3 patients with 18 -month medication showed disease progression. This difference was statistically significant as well.

Conclusions: Huaier granules could play an important role in post-surgical adjuvant therapy of TNBC patients, specially by effectively increasing the DFS and OS of breast cancer patients at middle to advanced stage.

Keywords: Huaier granules; breast cancer; traditional medicine; overall survival; disease-free survival (DFS)

Submitted Jul 26, 2019. Accepted for publication Nov 29, 2019.

doi: $10.21037 /$ gs.2019.12.08

View this article at: http://dx.doi.org/10.21037/gs.2019.12.08 


\section{Introduction}

Breast cancer has become one of the most common causes of cancer in women in China, accounting for $15 \%$ of all new malignant tumors as indicated by recent data, posing great threat to females' health and lives. What's more, it has the highest mortality of cancers in women aged over 45 years (1). Globally, the 5-year survival rate of female breast cancer patients at different stages is higher than $85 \%$. However, the rates in certain underdeveloped regions are much lower, such as Africa or India and China in Asia (2). Since Bernard Fisher proposed about 40 years ago that breast cancer was likely to be systemic at the time of diagnosis and represent potential metastases during the life of the host (3), the medical community has gradually come to recognize the importance of systemic therapy in breast cancer treatments. In particular, drug treatments after breast cancer surgery have been recognized as the main option for prevention of post-surgical recurrence and metastasis, and for the improvement of post-surgical survival rates in breast cancer patients.

Triple-negative breast cancer (TNBC) is one special molecular type featured with a negative profile for three molecular markers including ER, PR and HER2. TNBC is highly aggressive with poor prognosis and common for younger female population $(4,5)$, accounting for about $15-20 \%$ of all new breast cancer cases (6). It is usually belonged to the basal-like breast cancer (7). TNBC patients are generally young in age and have a family history of breast cancer, also bear larger sizes of mass and a higher probability of lymph node metastasis. Local recurrence and distant metastasis are commonly observed after surgery for TNBC, causing main reason for poor prognosis (8). In addition, endocrine therapy and targeted therapy cannot be administered after the completion of routine therapy in TNBC due to it lacks a therapeutic target (9). Hence, appropriate follow-up treatment for TNBC is an ongoing problem.

The developments in traditional Chinese medicine (TCM) with significant progress achieved in the field of cancer treatment provide potentials of TCM treating breast cancer. Huaier (Trametes robiniophila Murr.) has long been widely used in TCM as a medicinal fungus with history more than 1,600 years (10). A large number of clinical studies have shown that it has achieved good efficacy in prevention of tumor recurrence and metastasis $(11,12)$, and thus being able to effectively enhance patients' life qualities (13). In particular, Huaier granules have shown significant therapeutic efficacy, excellent safety, and minimal side effects in clinical studies of adjuvant therapy for liver cancer, lung cancer, breast cancer, and other solid tumors (10). Therefore, in order to further investigate the efficacy and safety of Huaier granules in post-surgical therapy for Stage I-III TNBC patients, we therefore conducted this randomised controlled trial (RCT) to observe its effects on the post-surgical safety and survival rates of TNBC patients.

\section{Methods}

The trial was registered at the Chinese Clinical Trial Registry before it started, the registration number is: ChiCTR-TRC-11001250.

\section{Inclusion and exclusion criteria}

A total of 201 TNBC patients were admitted to our hospital between October, 2010 to September, 2014. All patients underwent modified radical mastectomy were enrolled in this study within 1 year after surgery. Patients' postsurgical pathologic stages were stages I-III (American Joint Committee on Cancer, 7th edition). All patients were female, aged 26-70 years old, with Karnofsky Performance Status (KPS) score of $\geq 70$ points, ECOG score of 0 or 1 , no history of other malignant tumors, or any immune, cardiovascular, or chronic diseases. Patients were excluded with one of the conditions, including: (I) pregnancy or nursing; (II) comorbid severe heart, liver, kidney, and endocrine diseases, with less than 2 years expected survival time; (III) allergy to the studied drug; (IV) KPS score less than 60 points; (V) inability to receive treatment or attend follow-up visits on time for various reasons. All the patients had signed informed consents prior to recruitment (The approved No. of the ethic committee: KY201017).

Selected patients were randomly allocated to the experimental group (101 cases) or the control group (100 cases). Patients in both groups entered the adjuvant therapy stage within 2 weeks after surgery. Differences in age, menopausal status, tumor-node-metastasis (TNM) stage, lymph node metastasis, chemotherapy regimen, and other general information between the two groups were not statistically significant $(\mathrm{P}>0.05)$ (Table 1).

\section{Experimental methods}

Patients in the experimental group were treated with Huaier granules by orally taking $20 \mathrm{~g}$ each time with, 3 times a day. 
Table 1 Comparison of patients' general information $\mathrm{n}(\%)$

\begin{tabular}{|c|c|c|c|c|c|}
\hline Factor & $\mathrm{n}$ & Experimental group $(n=101)$ & Control group $(n=100)$ & $\chi^{2}$ & $\mathrm{P}$ \\
\hline \multicolumn{6}{|l|}{ Age (years) } \\
\hline$<45$ & 84 & $45(44.6)$ & $39(39.0)$ & 0.637 & 0.425 \\
\hline$\geq 45$ & 117 & $56(55.4)$ & $61(61.0)$ & & \\
\hline \multicolumn{6}{|l|}{ Menopausal status } \\
\hline Postmenopausal & 66 & $30(29.7)$ & $36(36.0)$ & & \\
\hline \multicolumn{6}{|l|}{ Lymph node states } \\
\hline Metastasis & 105 & $57(56.4)$ & $48(48.0)$ & 1.433 & 0.231 \\
\hline No metastasis & 96 & $44(43.6)$ & $52(52.0)$ & & \\
\hline Stage II & 101 & $49(48.5)$ & $52(52.0)$ & & \\
\hline Stage III & 58 & $32(31.7)$ & $26(26.0)$ & & \\
\hline \multicolumn{6}{|l|}{ Chemotherapy } \\
\hline None & 5 & $2(2.0)$ & $3(3.0)$ & 4.880 & 0.189 \\
\hline FEC/FAC & 8 & $7(6.9)$ & $1(1.0)$ & & \\
\hline TE & 129 & $62(61.4)$ & $67(67.0)$ & & \\
\hline TEC & 59 & $30(29.7)$ & $29(29.0)$ & & \\
\hline
\end{tabular}

FEC, fluorouracil, epirubicin, cyclophosphamide; FAC, fluorouracil, adriamycin, cyclophosphamide; TE, docetaxel, epirubicin; TEC, docetaxel, epirubicin, cyclophosphamide..

Medication was started during chemotherapy or at the time in 6 or 18 months after chemotherapy. The medication was continued regardless of any disease progression occurred during treatment. The control group did not use TCM preparations during this process. Neither of the groups received other antitumor drugs or immune enhancers (e.g., interleukin, interferon, etc.) during chemoradiotherapy.

All patients were followed up until July 31, 2017. The disease-free survival (DFS) and overall survival (OS) were measured as main outcome, and differences between experimental and control groups were compared. During the treatment and follow-up periods, both groups visited the hospital regularly for routine blood tests, repeated tests of liver function and kidney function, chest radiograph, and abdominal and breast ultrasound. Patients also underwent a bone scan and head computed tomography (CT) screening once a year. The time of occurrence for local recurrence or distant metastasis was recorded to calculate the DFS and OS. Disease progression was defined based on local recurrence, distant metastasis, or patient death.

This study was reviewed and approved before implementation by the ethics committee of Southwest Hospital affiliated to Third Military Medical University. This study has also been registered on Chinese Clinical Trial Register with registration number of ChiCTRTRC-11001250. All patients participated in the study voluntarily and gave informed consent in writing. During experimental process, patients had the right to withdraw from the study at any time without discrimination or penalty.

\section{Statistical analysis}

All data were analyzed using SPSS 17.0 statistical software. Measurement data were expressed using the mean \pm standard deviation $(\mathrm{x} \pm \mathrm{s})$. Count data were analyzed using the $\chi^{2}$ test. Kaplan-Meier survival analysis was performed on 

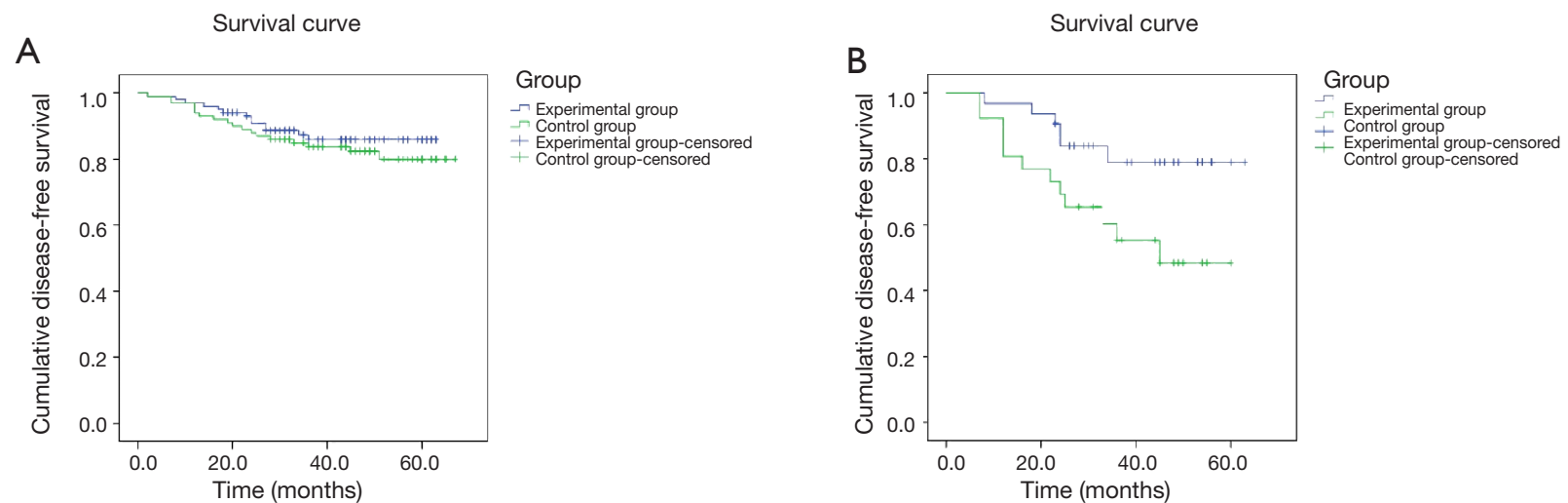

Figure 1 Disease-free survival curves in different groups. (A) Comparison of 5-year disease-free survival among all enrolled patients between the experimental and control groups; (B) comparison of 5-year disease-free survival among Stage III patients between the experimental and control groups.

DFS and OS. A two-tailed probability of $\mathrm{P}<0.05$ indicated the difference was statistically significant.

\section{Results}

\section{Post-chemotherapy survival and drug administration}

Thirteen out of 101 patients in the experimental group showed disease progression within 10 months after surgery, including 10 patients who died of disease progression except one died of abnormal causes. In the control group, 18 out of 100 patients showed disease progression within 7 months after surgery, including 14 patients who all died of disease progression. Disease progression was determined by local recurrence, manifested as chest wall recurrence and supraclavicular upper and lower lymph node metastasis, and distant metastasis, manifested as organ metastases in the lungs, bone, liver, and brain. No severe adverse reactions were observed in the experimental group when receiving the Huaier granules. Moreover, the drug had good palatability; hence, patient compliance was high.

\section{Effects of Huaier granules on the 5-year DFS of TNBC patients}

The median follow-up time was 46 months. 5-year DFS of the 100 patients in the control group was $82 \%$, and $87.1 \%$ for the 101 patients in experimental group; the difference between the 2 groups was not statistically significant. However, sub-group analysis of TNBC patients with different TNM stages indicated that 5-year DFS of stage III patients in the control group was $53.8 \%$, which was much lower than that of stage III patients in the experimental group of $81.3 \%$; this difference was statistically significant (Figure 1).

\section{Effects of Huaier granules on the 5-year OS of TNBC patients}

The 5 -year OS was $86 \%$ and $90.1 \%$ in control and experimental group respectively, indicating no significant difference. However, sub-group analysis of TNBC patients with different TNM stages indicated that 5-year OS of stage III patients in the control group was $65.4 \%$, much lower than that of stage III patients in the experimental group as $87.5 \%$; the difference was statistically significant (Table 2, Figure 2).

\section{Effects of Huaier granule treatment duration on the disease progression of TNBC patients}

The 101 TNBC patients in the experimental group were randomly divided into 2 groups: 49 patients received Huaier granules for 6 months and 52 patients received prolonged medication for 18 months. In the 6-month medication group, 10 patients showed disease progression, whereas in the 18-month medication group, only 3 patients showed disease progression. The difference between the 2 groups was statistically significant (Table 3).

\section{Discussion}

Breast cancer can be classified into different subtypes based 
Table 2 Comparison of survival between the two groups

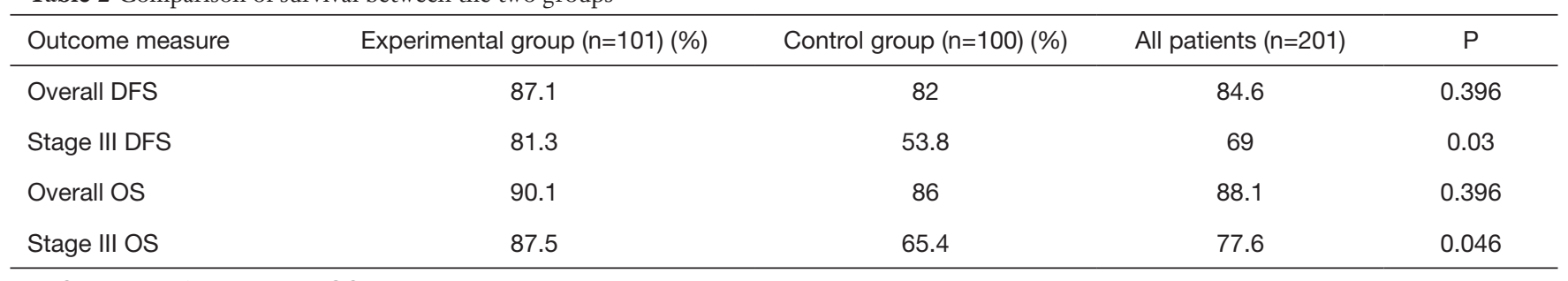

DFS, disease-free survival; OS, overall survival.
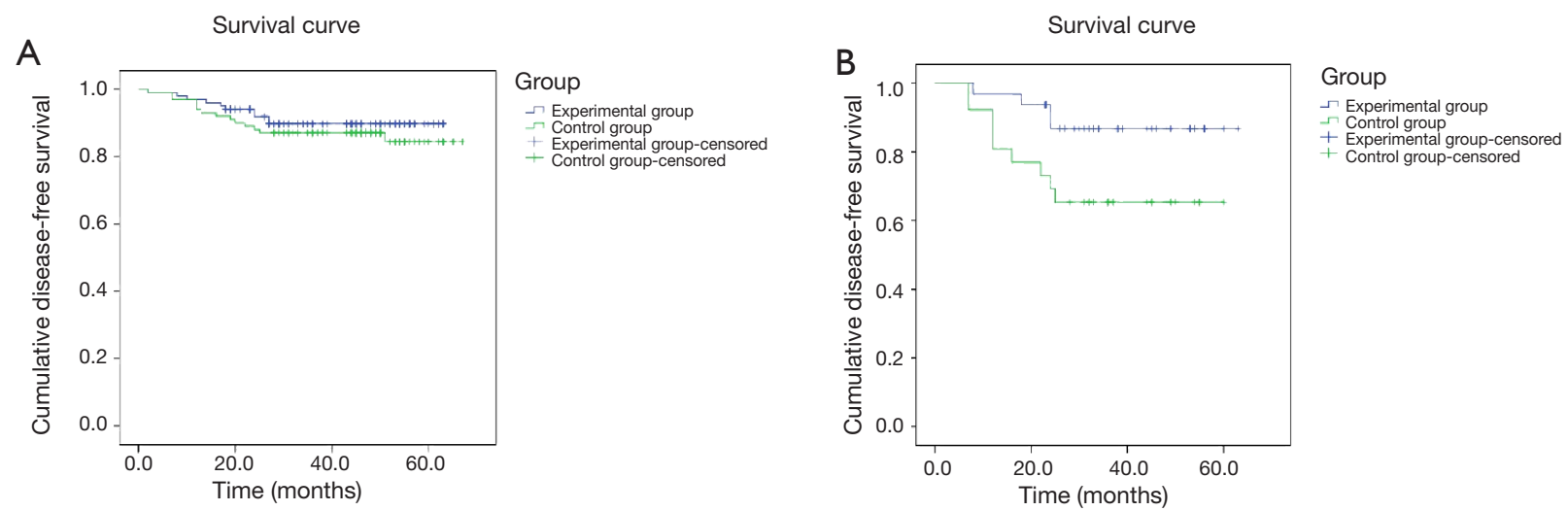

Figure 2 Five years over-all survival in different groups. (A) Comparison of 5-year overall survival among all enrolled patients between the experimental and control groups; (B) comparison of 5-year overall survival among Stage III patients between the experimental and control groups.

Table 3 Medication duration and disease progression in the experimental group, $\mathrm{n}$ (\%)

\begin{tabular}{lcccc}
\hline Medication duration & $\mathrm{n}$ & Disease progression $(\mathrm{n}=13)$ & No disease progression $(\mathrm{n}=88)$ & $\chi^{2}$ \\
\hline 6 months & 49 & $10(76.9)$ & $39(44.3)$ & 4.821 \\
18 months & 52 & $3(23.1)$ & $49(55.7)$ & 0.028 \\
\hline
\end{tabular}

on genetic markers, including basal-like, luminal A, luminal B, and human epidermal growth factor receptor 2 (HER-2)/neupositive breast cancers. These different subtypes of breast cancer have shown significant differences in treatment reactivity and prognosis $(14,15)$. TNBC and basal-like breast cancer (i.e., expression of basal cytokeratins: CK5/6, CK15, CK17 and/or epidermal growth factor receptor (EGFR) are similar subtypes known by features of unstable gene copy number, proneness to mutations, and large numbers of single nucleotide polymorphisms. Moreover, lack of heterozygosity would suggest high susceptibility to DNA damage. Furthermore, TNBC often shows BRCA1 mutations and high MAPK expression to a certain extent. Patients with high MAPK expression frequently show anthracycline resistance, high rates of tumor recurrence, and a short survival period. About $80-90 \%$ of TNBC cases are basal-like breast cancers, which are characterized by highly aggressive nature and poor prognosis, along with high level of invasiveness and probability of lymph node metastasis. Besides, local recurrence and distant metastasis are highly possible resulting in low OS. It has been reported that the OS and DFS of TNBC patients are significantly lower than that of patients with other types of breast cancer, and this difference is particularly prominent among patients at middle to advanced stages of breast cancer. However, the sensitivity of TNBC to chemotherapy drugs is not any lower than that of other subtypes, and may even be higher $(16,17)$. Usually, only chemotherapy is used to treat TNBC 
due to lacking of opportunities for endocrine and targeted therapy, Therefore, the primary reasons for the poor prognosis of TNBC might be related to its highly aggressive nature, lacking of effective risk reduction measures, and its biological characteristics (18).

Endocrine therapy plays a crucial role in post-surgical adjuvant therapy of hormone-dependent recurrent and metastatic breast cancer, and early-stage breast cancer. Nevertheless, common practice under modern medicine for hormone-independent early-stage breast cancer [i.e., estrogen- (ER) and progesterone-receptor (PR) negative disease] suggests only follow-ups and observations with no further sequential therapy after surgery, chemotherapy or radiotherapy. In contrast, TCM involves syndrome differentiation based on the effects of physical condition, age, personality, past treatments, and other perspectives. Its emphasis is on regulating and modifying the basis of tumor production (i.e., deficiency in origin and excess in superficiality) to prevent local recurrence and distant metastasis. In recent years, numerous studies have shown that TCM can inhibit the migration, proliferation, and apoptosis of tumor cells, and strengthen the patient's immune system, thus improving the patient's quality of life, and reducing the adverse reactions caused by surgery, chemotherapy, and other damaging treatments $(19,20)$. Furthermore, TCM has limited toxic side effects and low costs. Therefore, the identification of natural active substances in TCM for the post-surgical adjuvant treatment of TNBC is of important scientific value and clinical significance in the prevention of recurrence and metastasis.

Huaier refers to the dried fruiting bodies of the Trametes robiniophila Murr. fungus from the Polyporaceae family, and its main active ingredients are proteoglycans (PS-T)(10). A large number of clinical studies have shown that it has achieved good efficacy in the prevention and treatment of tumor recurrence and metastasis, while also is able to effectively enhance patients' quality of life. In addition, Huaier has shown multiple effects in the comprehensive treatment of breast cancer, including anti-tumor activity, reversal of drug resistance, and immunomodulation. In clinical studies using Huaier granules to treat middle-stage to advanced primary liver cancer in elderly patients, the results have shown that Huaier granules could effectively stabilize the tumor, reduce alpha fetoprotein (AFP), improve liver function, or reduce carcinoembryonic antigen (CEA) levels, while also improve the patient's quality of life (21). Huaier can also improve T-cell function in patients with
Stage III-IV non-small cell lung cancer. Hence, it can be used as a comprehensive treatment measure for non-small cell lung cancer, and improve patients' long-term survival (22). Further experimental studies have shown that Huaier granules could prevent and treat tumor recurrence and metastasis by strengthening the body's immune function, promoting the apoptosis of tumor cells, enhancing sensitivity to chemotherapy drugs, reversing drug resistance, and resisting tumor angiogenesis $(11,23)$. With regard to the use of Huaier granules in comprehensive breast cancer treatments, some clinical studies have found that the granules could reduce toxicity, enhance efficacy, and improve the patients' immune functions in all phases of comprehensive breast cancer treatments. Further experimental studies have indicated that it might play a positive role in prevention and treatment of breast cancer recurrence and metastasis (24). In addition, Huaier extract is a novel ER modulator and is a promising drug for prevention and treatment of ER $\alpha$ positive human breast cancers (25). Studies examining the regulatory effects of Huaier granules on the post-surgical immune function of breast cancer patients found that Huaier granules could increase the patients' lymphocyte count, and IgA, IgG, and IgM values. They are also able to facilitate the recovery of post-surgical humoral immune function and cellular immune function in breast cancer patients $(26,27)$. Numerous experimental studies have shown that Huaier granules have multiple effects, such as angiogenesis inhibition, direct apoptosis in cells, comprehensive immunomodulatory functions, and significant inhibitory effects on high-metastatic tumor cells (28). These effects are essential for the prevention and treatment of tumor recurrence and metastasis.

This study demonstrates that Huaier granules play an important role in the post-surgical adjuvant therapy of TNBC patients, especially be able to effectively increase the DFS and OS of breast cancer patients at middle to advanced stage. Furthermore, prolonging the medication duration could effectively reduce the possibility of disease progression, implying the potential of improving postsurgical quality of life and long-term prognosis of middlestage to advanced TNBC patients. However, further investigations are needed on the clarifying the underlying molecular mechanisms of this phenomenon.

\section{Acknowledgments}

None. 


\section{Footnote}

Conflicts of Interest: The authors have no conflicts of interest to declare.

Ethical Statement: The authors are accountable for all aspects of the work in ensuring that questions related to the accuracy or integrity of any part of the work are appropriately investigated and resolved. The study was approved by the Ethics Committee of Southwest Hospital affiliated to Third Military Medical University (KY201017) and written informed consent was obtained from all patients.

\section{References}

1. Chen W, Zheng R, Baade PD, et al. Cancer statistics in China, 2015. CA Cancer J Clin 2016;66:115-32.

2. Allemani C, Weir HK, Carreira H, et al. Global surveillance of cancer survival 1995-2009: analysis of individual data for $25,676,887$ patients from 279 population-based registries in 67 countries (CONCORD-2). Lancet 2015;385:977-1010.

3. Fisher B, Wolmark N, Redmond C, et al. Findings from NSABP Protocol No. B-04: comparison of radical mastectomy with alternative treatments. II. The clinical and biologic significance of medial-central breast cancers. Cancer 1981;48:1863-72.

4. Badve S, Dabbs DJ, Schnitt SJ, et al. Basal-like and triplenegative breast cancers: a critical review with an emphasis on the implications for pathologists and oncologists. Mod Pathol 2011;24:157-67.

5. Tariq K, Farhangi A, Rana F. TNBC vs non-TNBC: A retrospective review of differences in mean age, family history, smoking history, and stage at diagnosis. Clin Adv Hematol Oncol 2014;12:377-81.

6. Wong-Brown MW, Meldrum CJ, Carpenter JE, et al. Prevalence of BRCA1 and BRCA2 germline mutations in patients with triple-negative breast cancer. Breast Cancer Res Treat 2015;150:71-80.

7. Foulkes WD, Smith IE, Reis-Filho JS. Triple-negative breast cancer. N Engl J Med 2010;363:1938-48.

8. Elsawaf Z, Sinn HP. Triple-Negative Breast Cancer: Clinical and Histological Correlations. Breast Care (Basel) 2011;6:273-8.

9. O'Reilly EA, Gubbins L, Sharma S, et al. The fate of chemoresistance in triple negative breast cancer (TNBC). BBA Clin 2015;3:257-75.
10. Song X, Li Y, Zhang H, et al. The anticancer effect of Huaier (Review). Oncol Rep 2015;34:12-21.

11. Zheng J, Li C, $\mathrm{Wu} \mathrm{X}$, et al. Huaier polysaccharides suppresses hepatocarcinoma MHCC97-H cell metastasis via inactivation of EMT and AEG-1 pathway. Int J Biol Macromol 2014;64:106-10.

12. Yan $X$, Lyu $T$, Jia N, et al. Huaier aqueous extract inhibits ovarian cancer cell motility via the AKT/GSK3beta/betacatenin pathway. PLoS One 2013;8:e63731.

13. Zhang N, Kong X, Yan S, et al. Huaier aqueous extract inhibits proliferation of breast cancer cells by inducing apoptosis. Cancer Sci 2010;101:2375-83.

14. Sotiriou C, Neo SY, McShane LM, et al. Breast cancer classification and prognosis based on gene expression profiles from a population-based study. Proc Natl Acad Sci U S A 2003;100:10393-8.

15. Rakha EA, El-Sayed ME, Green AR, et al. Prognostic markers in triple-negative breast cancer. Cancer 2007;109:25-32.

16. Banerjee S, Reis-Filho JS, Ashley S, et al. Basal-like breast carcinomas: clinical outcome and response to chemotherapy. J Clin Pathol 2006;59:729-35.

17. Carey LA, Dees EC, Sawyer L, et al. The triple negative paradox: primary tumor chemosensitivity of breast cancer subtypes. Clin Cancer Res 2007;13:2329-34.

18. Chu KC, Lamar CA, Freeman HP. Racial disparities in breast carcinoma survival rates: seperating factors that affect diagnosis from factors that affect treatment. Cancer 2003;97:2853-60.

19. Li C, Wu X, Zhang H, et al. A Huaier polysaccharide inhibits hepatocellular carcinoma growth and metastasis. Tumour Biol 2015;36:1739-45.

20. Liu J, Lau EY, Chen J, et al. Polysaccharopeptide enhanced the anti-cancer effect of gamma-tocotrienol through activation of AMPK. BMC Complement Altern Med 2014;14:303.

21. Lei JY, Yan LN, Zhu JQ, et al. Hepatocellular Carcinoma Patients May Benefit From Postoperative Huaier Aqueous Extract After Liver Transplantation. Transplant Proc 2015;47:2920-4.

22. Zheng J, Li C, Wu X, et al. Astrocyte elevated gene1 (AEG-1) shRNA sensitizes Huaier polysaccharide (HP)-induced anti-metastatic potency via inactivating downstream P13K/Akt pathway as well as augmenting cell-mediated immune response. Tumour Biol 2014;35:4219-24.

23. Li C, Wu X, Zhang H, et al. A Huaier polysaccharide restrains hepatocellular carcinoma growth and metastasis 
by suppression angiogenesis. Int J Biol Macromol 2015;75:115-20.

24. Kong X, Ding X, Yang Q. Identification of multitarget effects of Huaier aqueous extract via microarray profiling in triple-negative breast cancer cells. Int J Oncol 2015;46:2047-56.

25. Wang X, Zhang N, Huo Q, et al. Huaier aqueous extract suppresses human breast cancer cell proliferation through inhibition of estrogen receptor alpha signaling. Int J Oncol 2013;43:321-8.

Cite this article as: Wang M, Hu Y, Hou L, Pan Q, Tang P, Jiang J. A clinical study on the use of Huaier granules in postsurgical treatment of triple-negative breast cancer. Gland Surg 2019;8(6):758-765. doi: 10.21037/gs.2019.12.08
26. Li Y, Qi W, Song X, et al. Huaier extract suppresses breast cancer via regulating tumor-associated macrophages. Sci Rep 2016;6:20049.

27. Wang X, Zhang N, Huo Q, et al. Huaier aqueous extract inhibits stem-like characteristics of MCF7 breast cancer cells via inactivation of hedgehog pathway. Tumour Biol 2014;35:10805-13.

28. Wang X, Zhang N, Huo Q, et al. Anti-angiogenic and antitumor activities of Huaier aqueous extract. Oncol Rep 2012;28:1167-75. 